\title{
Towards a causative function of histone modifications in regulation of chromatin dynamics and transcription
}

\author{
Philipp Tropberger ${ }^{1}$, Sebastian Pott ${ }^{2,5}$, Kinga Kamieniarz ${ }^{1}$, Matthieu Caron ${ }^{3}$, Edison T Liư ${ }^{2,6}$, Raphael Margueron ${ }^{3}$, \\ Robert Schneider ${ }^{1,4^{*}}$
}

From Epigenetics and Chromatin: Interactions and processes

Boston, MA, USA. 11-13 March 2013

\section{Background}

The repertoire of known histone modifications is far from complete. Most attention has been focused on the $\mathrm{N}$-terminal histone tails. However, modifications on the lateral surface of the histone octamer can - potentially directly regulate nucleosome dynamics. Because of their structurally important position close to the DNA, we can understand what these modifications are actually doing to nucleosome structure and/or dynamics.

\section{Results}

We identified H3K122, a residue at the lateral surface, as a novel acetylation site and use it as a model to study the mechanism of action of lateral surface modifications. By applying a genome-wide approach we find that H3K122ac defines genetic elements associated with transcription and that it co-occurs with active chromatin marks. In line with this H3K122ac is catalyzed by transcriptional co-activators and induced by estrogen receptor signaling. To investigate if H3K122ac not only correlates with active transcription, but has a causative function, we developed an in vitro protocol to transcribe chromatin with defined acetylations. We show that chromatin with H3K122ac is transcribed more efficiently than chromatin with unmodified $\mathrm{H} 3$, or acetylated at the $\mathrm{H} 3$ tail, demonstrating that H3K122ac is capable of stimulating transcription. We show that this is through the relaxation of histone-DNA binding introduced by H3K122ac and the stimulation of nucleosome eviction by H3K122ac. This strongly supports our model that nucleosome function is directly regulated by specific lateral surface modifications.

${ }^{1}$ Max Planck Institute of Immunobiology and Epigenetics, 79108 Freiburg Germany

Full list of author information is available at the end of the article

\section{Conclusions}

Together our findings show that modifications in the nucleosomal core - such as H3K122ac - have a direct role in regulating chromatin function. We demonstrate that one acetylation is sufficient to stimulate transcription by modulating histone-DNA binding. This suggests that transcriptional regulators elicit their effects not only via signaling to histone-tails, but also via direct structural perturbation of nucleosomes by directing acetylation to the lateral octamer surface.

\section{Author details \\ ${ }^{1}$ Max Planck Institute of Immunobiology and Epigenetics, 79108 Freiburg, Germany. ${ }^{2}$ Cancer Biology and Pharmacology, Genome Institute of Singapore, 138672 Singapore. ${ }^{3}$ Institut Curie MR 3215 CNRS, U934INSERM, 26, rue d'Ulm, 75005 Paris, France. ${ }^{4}$ Institut de Génétique et de Biologic Moléculaire et Cellulaire, CNRS UMR 7104, INSERM U 964, Université de Strasbourg, 67404 Illkirch, France. ${ }^{5}$ Present address: Department of Biology, University of North Carolina at Chapel Hill, NC 27599-3280, USA. ${ }^{6}$ Present address: The Jackson Laboratory, 600 Main Street, Bar Harbor, Maine, 04609, USA.}

Published: 18 March 2013

doi:10.1186/1756-8935-6-S1-030

Cite this article as: Tropberger et al.: Towards a causative function of histone modifications in regulation of chromatin dynamics and transcription. Epigenetics \& Chromatin 2013 6(Suppl 1):O30. 